\title{
Anchor Limited $Q$ in Flexural Mode Resonators
}

\author{
Joshua E.-Y. Lee, Jize Yan, and Ashwin A. Seshia \\ Department of Engineering \\ University of Cambridge \\ Cambridge, United Kingdom \\ Email: joshlee@cantab.net
}

\begin{abstract}
This paper reports a preliminary examination of the effect of anchor geometry design on the quality factor of flexural mode resonators operating in vacuum using both $\mathrm{FE}$ simulation and measurements of resonator frequency response. Three types of structures have been considered in this study: an elliptical mode ring, a double ended tuning fork, and a doubly-clamped beam. We consider the relative distribution of strain energies in both the resonant structure and the connecting stem, which is indicative of the measured quality factor. The measured quality factors of the different structures are compared against each other, based on which suggestions are proposed for optimizing the anchor limited quality factor $(Q)$ in flexural mode micromechanical resonators.
\end{abstract}

Keywords-quality factor; clamping loss; energy dissipation; micromechanical resonators;

\section{INTRODUCTION}

Silicon micromechanical resonators have been the focus of much recent interest as a possible alternative to quartz, which have been traditionally favored as the time-keeping element for a diverse range of applications [1]. As a material for realizing micromechanical resonators, silicon exhibits excellent scaling characteristics, including the benefit of potentially integrating with standard IC electronics. As a mechanical material, silicon also possesses excellent properties [2], and has been shown to be capable of realizing micromechanical resonators of high quality factors $(Q)$ (as high as over a million) [3]. A high $Q$ is desirable as it leads to improved stability of an oscillator and lowers the close-to-carrier phase noise.

The $Q$ of a resonant system is defined by the ratio of the total energy stored to the energy dissipated per cycle during resonance:

$$
Q=2 \pi(\text { Energy stored / Energy dissipated per cycle) }
$$

The $Q$ is therefore enhanced when the net dissipation in the system is reduced in proportion to the total energy stored. Common sources of energy dissipation in microresonators include surface loss, thermoelastic dissipation (TED), viscous damping, and clamping loss. The $Q$ of a resonator is ultimately determined by the sum of all sources of energy dissipation. In vacuum, the effect of viscous damping is reduced sufficiently such that TED and clamping loss become the dominant sources of energy dissipation.

This work was supported by the U.S. Army Soldier Systems Centre.
This paper focuses on the clamping loss in a microresonator and the effect on the resonator $Q$. During resonance, a fraction of the energy stored in the resonant microstructure may be transmitted through the suspension stems and fixed anchors to the substrate and surrounding environment - this is the source of clamping loss. In this paper, three structural topologies have been considered:

(1) Elliptical mode ring resonator

(2) Clamped-free double ended tuning fork resonator

(3) Doubly-clamped beam resonator

For the case of the ring resonator, two stem geometries are examined with the aim of enhancing $Q$, namely a straight stem and a T-shaped stem. The impact of both geometries on $Q$ is studied using FE modeling by considering the distribution of strain energies in the structure and stem. This is followed by experimental measurements of the same modeled structures. The $Q$ measurements for a clamped-free double ended tuning fork driven in-phase and anti-phase, as well as results from two doubly-clamped beams, are presented thereafter. Comparisons between the measured data from each structure are indicative of a significant impact on $Q$ in flexural mode resonators through de-coupling the anchors from the resonant structure. The devices reported herein have been fabricated in a foundry SOI micromachining process $(25 \mu \mathrm{m}$ thick) by MEMSCAP.

\section{MODELING AND ANALYSIS}

\section{A. Stem Geometry - Elliptical mode ring resonator}

The anchor limited $Q$ of a resonator may be approximated by the ratio of the maximum total strain energy (which occurs at maximum deflection) in the system relative to the energy lost through the anchors. For a ring structure, as shown in Fig. 1, the suspended ring is supported through stems located on the perimeter of the structure which are connected to the anchors. A fraction of strain energy stored in these stems is lost during vibration, thus the anchor limited $Q$ is dependent on the ratio of the strain energies in the structure and stem:

$$
\begin{gathered}
Q_{\text {anchor }}=f(\Gamma) \\
\Gamma=E_{S} / E_{A}
\end{gathered}
$$


$E_{S}$ and $E_{A}$ denote the maximum strain energies in the structure and stem respectively. Therefore, if the strain energy stored in the stem is larger in proportion to that of structure, the effect of anchor related losses would be comparatively more dominant. The relative values of the strain energies for both stem designs (while keeping the dimensions of the ring the same) can be calculated and compared using FEA simulation, as shown in Fig. 2, simulated for the elliptical mode. The dimensions of the structure and annexed stems are summarized in the Table I. The dimensions of the straight stem are identical to those of the T-shaped stem's central extension.
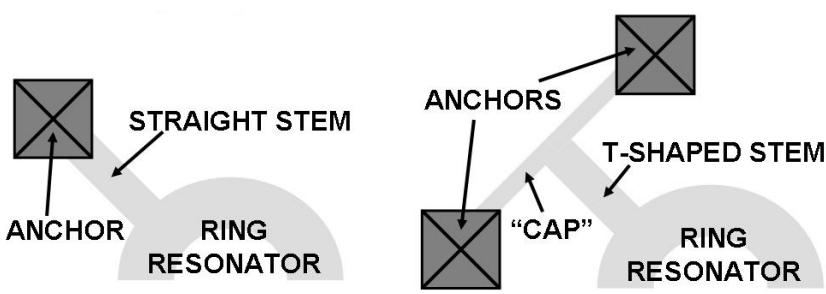

Figure 1. Stick diagrams illustrating the arrangement of two stem designs, (a) straight and (b) T-shaped, in relation to the ring resonator
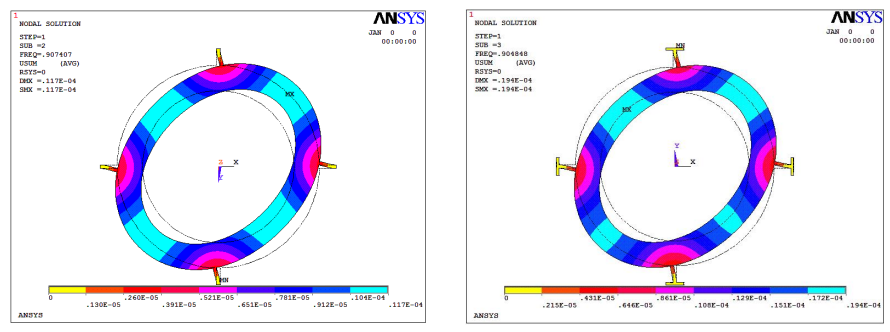

Figure 2. Elliptical mode shape of a ring resonator with (a) straight connecting anchoring stem, (b) T-shaped connecting anchoring stem

TABLE I. DIMENSIONS OF RING RESONATOR WITH ANNEXED STEMS

\begin{tabular}{|l|c|}
\hline Parameter (units) & Value \\
\hline Inner radius of the ring structure $(\mu \mathrm{m})$ & 300 \\
\hline Outer radius of the ring structure $(\mu \mathrm{m})$ & 400 \\
\hline Length of "cap" for the T-shaped stem $(\mu \mathrm{m})$ & 70.7 \\
\hline Width of "cap" for the T-shaped stem $(\mu \mathrm{m})$ & 10.6 \\
\hline Length of straight stem $(\mu \mathrm{m})$ & 67.2 \\
\hline Width of straight stem $(\mu \mathrm{m})$ & 14.1 \\
\hline
\end{tabular}

Table II summarizes the calculated relative distribution of strain energies of the stem and the annexed ring structure. For the T-stem, only the "cap" of the stem is used to calculate the strain energy in the stem. The calculated values of $\Gamma$ are about 5 times larger in the case of a T-stem compared to the straight stem. Both values are also much smaller by 50 times relative to the values of $\Gamma$ calculated for a bulk mode disk resonator of comparable dimensions, given in Table III, which we have reported elsewhere in [4]. It was observed in [4] that the stem topologies did not have a significant effect on the $Q$ of the disk, wherein a $Q$ of 2 million was measured. The calculated values of $\Gamma$ for the elliptical mode ring structures thus indicate, that in contrast to an elliptical mode disk structure, anchor related loss could be dominant. In the case where $Q$ is limited by anchor loss, we could expect the measured values of $Q$ to differ by the same margin (approximately 5 times) between a ring resonator connected to a T-stem and straight stem.
TABLE II. FEA COMPUTED RELATIVE STRAIN ENERGIES FOR ELLIPTICAL MODE (RING) MICRORESONATORS WITH DIFFERING STEM GEOMETRY [4]

\begin{tabular}{|c|c|c|}
\hline & Straight stem & T-shaped stem \\
\hline Structure $\left(\boldsymbol{E}_{\boldsymbol{S}}\right)$ & 15.7 & 15.7 \\
\hline Anchor-stem $\left(\boldsymbol{E}_{\boldsymbol{A}}\right)$ & 0.3 & 0.07 \\
\hline $\boldsymbol{\Gamma}=\boldsymbol{E}_{\boldsymbol{S}} / \boldsymbol{E}_{\boldsymbol{A}}$ & 52.3 & 224.3 \\
\hline
\end{tabular}

TABLE III. FEA COMPUTED RELATIVE STRAIN ENERGIES FOR ELLIPTICAL MODE (DISK) MICRORESONATORS WITH DIFFERING STEM GEOMETRY

\begin{tabular}{|c|c|c|}
\hline & Straight stem & T-shaped stem \\
\hline Structure $\left(\boldsymbol{E}_{\boldsymbol{S}}\right)$ & 583.3 & 583.3 \\
\hline Anchor-stem $\left(\boldsymbol{E}_{\boldsymbol{A}}\right)$ & 0.1857 & 0.06366 \\
\hline $\boldsymbol{\Gamma}=\boldsymbol{E}_{\boldsymbol{S}} / \boldsymbol{E}_{\boldsymbol{A}}$ & 3140 & 9160 \\
\hline
\end{tabular}

\section{B. Clamped-free double ended tuning fork}

Since the FEA simulation results from the previous section indicate a potential significant enhancement in $Q$ through decoupling the resonator from the anchors, a clamped-free double ended tuning fork (DETF) may be used to further evaluate the impact of de-coupling the resonator from the anchors. As depicted in Fig. 3, the DETF could be excited in 2 possible modes, described by the tines either moving in-phase or antiphase. The anti-phase mode possesses a slightly higher $Q$ over the in-phase mode due to mutual cancellation of stress waves at the anchors [5].
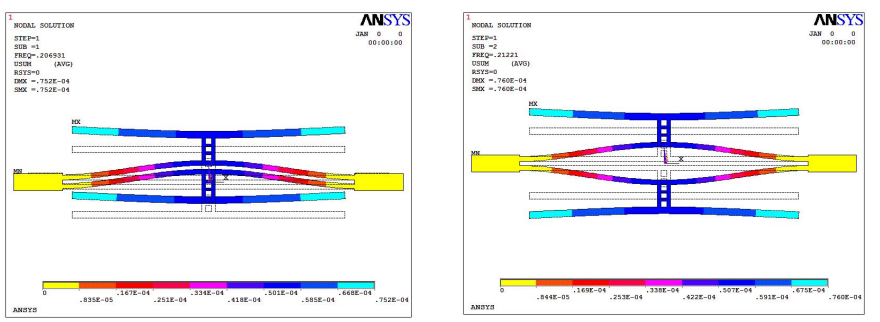

Figure 3. Mode shapes of a clamped-guided double ended tuning fork microresonator excited in the (a) in-phase mode, (b) anti-phase mode

\section{Doubly-clamped beam}

Finally, a doubly-clamped beam is considered as reference for comparisons with the DETF and ring structures. A DETF with the tines moving in-phase may be compared to 2 doublyclamped beams. Since both ends of the beam are clamped, the $Q$ of resonator would potentially be lower, due to higher loss.

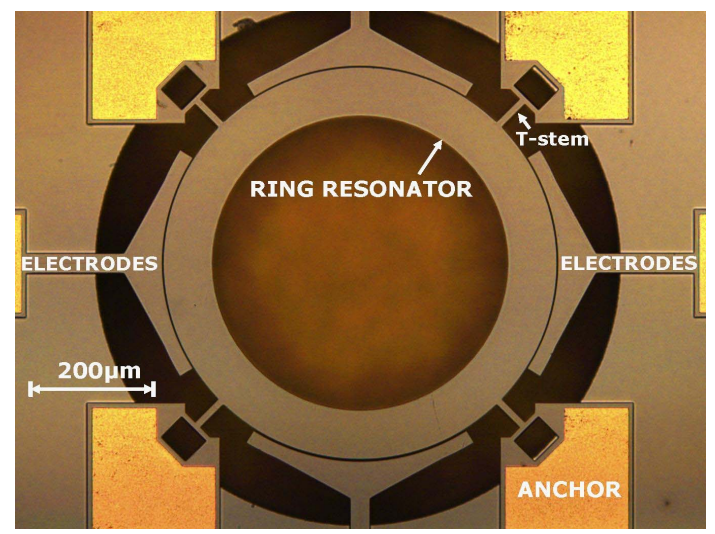

Figure 4. Micrograph of the fabricated SOI ring microresonator (T-stem) 


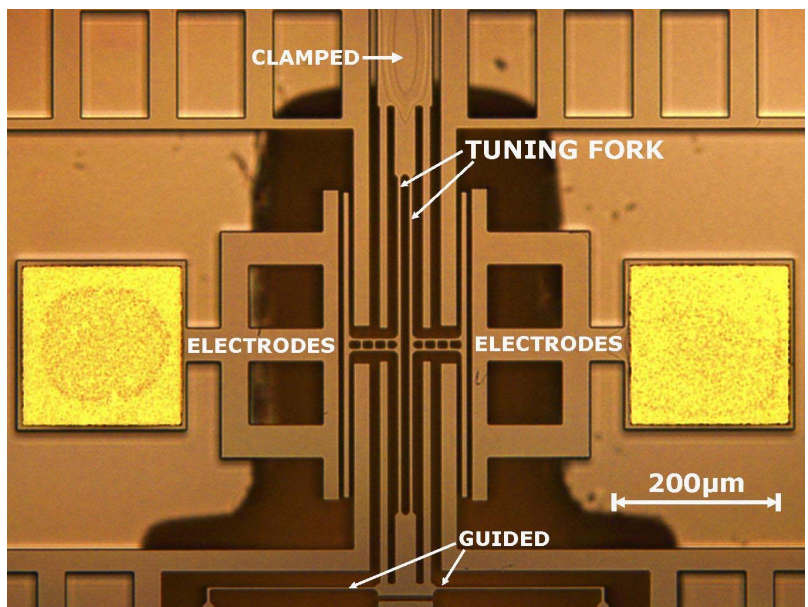

Figure 5. Micrograph of the fabricated SOI double ended fork resonator

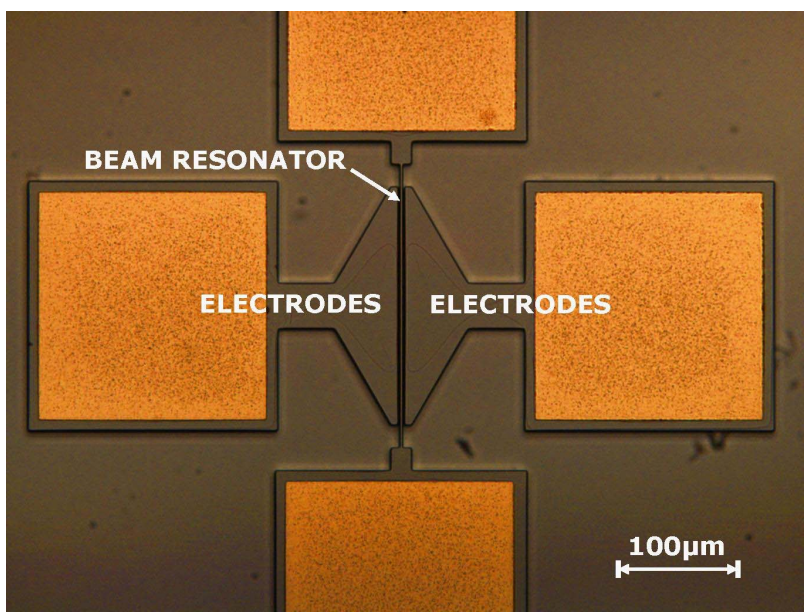

Figure 6. Micrograph of the fabricated SOI doubly-clamped beam resonator

\section{EXPERIMENT}

Figs. 4 to 6 each show optical micrographs of the fabricated resonators. All the resonators have been characterized inside a custom-built vacuum chamber at pressures below 10mTorr. The electrical transmission of each device was measured using a network analyzer (Agilent 4396B). The same set of voltages has been applied to both ring resonators. The voltages have also been kept the same while driving the DETF in-phase and out-of-phase. The electrical transmission of 2 doubly-clamped beams, fabricated on the same die, was measured similarly using identical voltages applied to each resonator. $Q$ values have been extracted from measurements of the transmission using the procedure described in [6].

The measured transmission for the ring structures is shown in Fig. 7 (straight) and Fig. 8 (T-shaped). Figs. 9 and 10 show the measured transmission for the same DETF, excited in the in-phase and anti-phase resonant modes respectively. The shape of curve in Fig. 9 arises from the phase-inversion of the motional current. Shown in Fig. 11 is the measured electrical transmission for one of the two doubly-clamped beams.

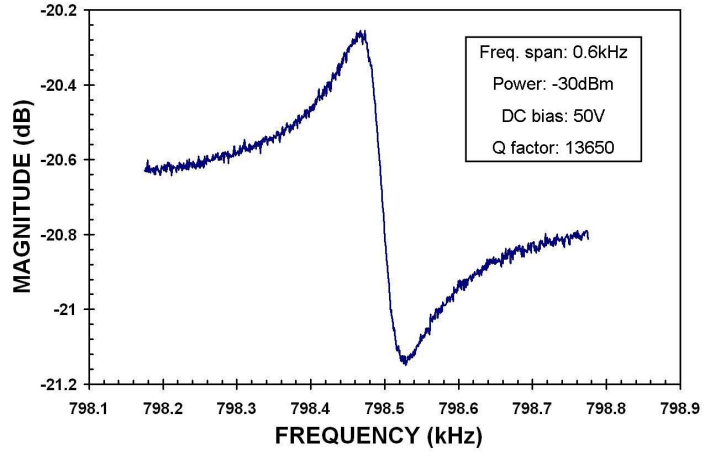

Figure 7. Measured electrical transmission of an elliptical mode ring microresonator connected through straight stems

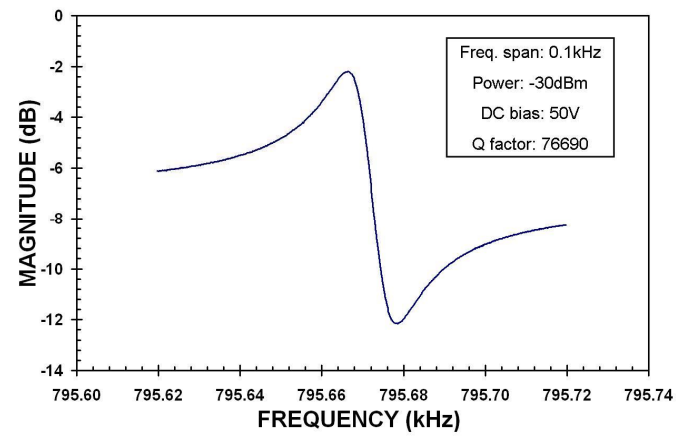

Figure 8. Measured electrical transmission of an elliptical mode ring microresonator connected through T-shaped stems

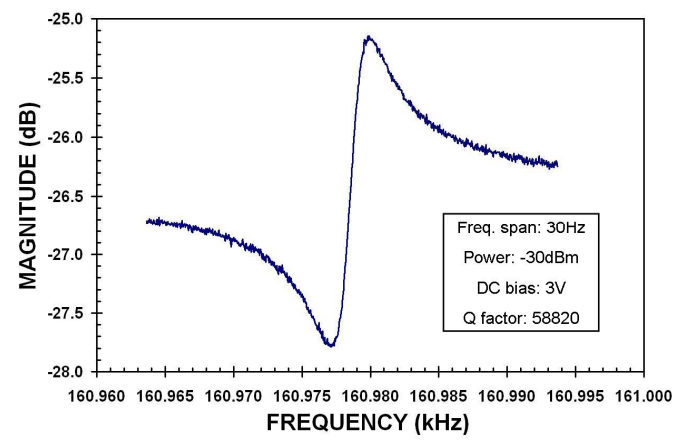

Figure 9. Measured electrical transmission of a double-ended tuning fork microresonator excited in the in-phase mode

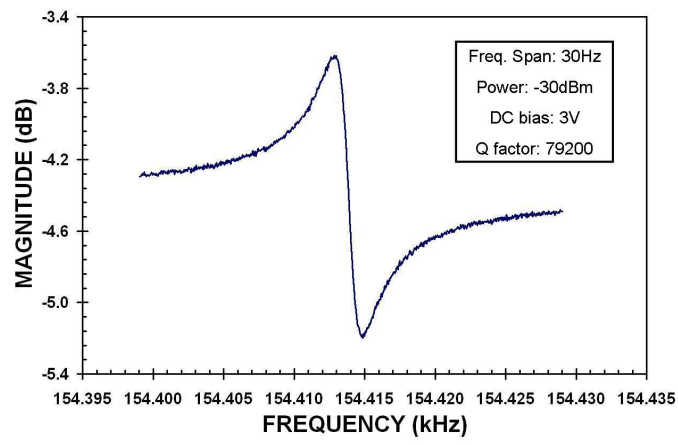

Figure 10. Measured electrical transmission of the double-ended tuning fork microresonator excited in the anti-phase mode 


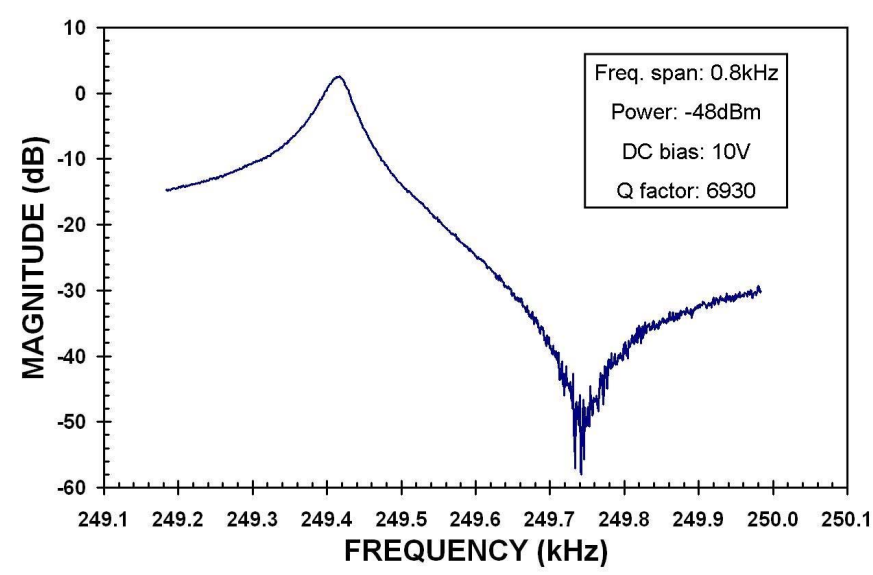

Figure 11. Measured electrical transmission of a doubly-clamped beam

\section{DISCUSSION}

\section{A. Impact of de-coupling}

The measured values of $Q$ for each device are summarized in Table IV. It may be seen that for the ring structure, the $Q$ associated with a T-shaped stem is just over 5 times that for a straight stem, which agrees with the prediction in Section II based on FEA simulation. As previously highlighted, this result indicates that the $Q$ for the elliptical mode ring resonator is limited by anchor related losses when a straight stem is used. This suggests that de-coupling the resonant structure from the anchors by using a T-shaped stem can be effective in reducing energy loss through the anchors and connecting stems.

\section{B. Comparison with no de-coupling}

In comparison to the in-phase mode of the clamped-free DETF, both the doubly-clamped beams characterized show a substantially lower $Q$ by almost an order of magnitude. Even though the dimensions of the doubly-clamped beam and the tines of the DETF differ, the significant disparity in $Q$ is still indicative of $Q$ enhancement through de-coupling one end of the tines from the anchors using suspended stems.

Finally, the measured $Q$ of the in-phase mode for the DETF is slightly lower than the anti-phase mode due to the mutual cancellation of stress waves meeting at the anchors.

\section{Energy storage capacitor}

As highlighted previously from Tables II and Table III, the $\Gamma$ ratios calculated for a bulk mode disk resonator are significantly higher than for flexural mode ring resonator. A comparison of calculated relative strain energies for a bulk mode disk resonator and the flexural mode ring resonator reveals that the higher values of $Q$ observed in the bulk mode could be attributed to their larger energy storage capacity due to a significantly larger stiffness. Therefore in the case of the bulk mode disk, the stem topology has little effect on $Q$, which is not the case for the flexural mode ring resonator, as shown in this paper.

TABLE IV. MEASURED Q FACTORS OF FABRICATED SOI RESONATORS

\begin{tabular}{|c|c|c|}
\hline \multirow{2}{*}{} & \multicolumn{2}{|c|}{ Elliptical mode ring } \\
\cline { 2 - 3 } & Straight stem & T-shaped stem \\
\hline \multirow{2}{*}{ Measured Q } & 13600 & 76700 \\
\hline & \multicolumn{2}{|c|}{ Clamped-free double ended tuning fork } \\
\cline { 2 - 3 } & In-phase mode & Anti-phase mode \\
\hline Measured Q & 58800 & 79200 \\
\hline \hline & \multicolumn{2}{|c|}{ Doubly-clamped beams } \\
\cline { 2 - 3 } & Beam A & Beam B \\
\hline Measured Q & 6040 & 6920 \\
\hline
\end{tabular}

\section{Conclusions}

We have shown in this paper that the quality factor in flexural mode resonators could be enhanced through decoupling the resonant structure from the anchors with the use of connecting stems. This conclusion has been observed through a study of three microstructures fabricated in SOI, each of which have been excited in a number of flexural modes. The observed trends from measurements on fabricated devices agree well with the FEA simulation and analytical predictions. The impact of anchor related loss on the quality factor of a resonator has been evaluated by considering the distribution of strain energy in the resonant structure and connecting stems.

\section{REFERENCES}

[1] C. T.-C. Nguyen, "MEMS technology for timing and frequency control," IEEE Trans. Ultron. Ferroelect. Freq. Contr., vol. 54, no. 1, pp. 251-270, Feb. 2007.

[2] K. E. Petersen, "Silicon as a mechanical material," Proc. IEEE, vol. 70, no. 5, pp. 420-457, May 1982.

[3] J. E.-Y. Lee, B. Bahreyni, Y. Zhu, and A. A. Seshia, "A single crystal silicon bulk acoustic mode microresonator oscillator," IEEE Elect. Dev. Lett., vol. 29, no. 7, pp. 701-703, Jul. 2008.

[4] J. E.-Y. Lee, J. Yan, and A. A. Seshia, "Quality factor enhancement of bulk acoustic mode resonators through anchor geometry design," Proc. EUROSENSORS XXII, Sep. 2008.

[5] A. A. Seshia, "Integrated micromechanical resonant sensors for inertial measurement systems," Ph.D. thesis, UC Berkeley, 2002.

[6] J. E.-Y. Lee, Y. Zhu, and A. A. Seshia, "Bulk acoustic mode single crystal microresonator with high quality factor," J. Micromech. Microeng., vol. 18, 064001, Jun. 2008. 